TOKYO J. Math.

VoL. 2, No. 1, 1979

\title{
Minimal Immersions of Riemannian Products into Real Space Forms
}

\author{
Norio EJIRI \\ Tokyo Metropolitan University \\ (Communicated by S. Yano)
}

\section{Introduction}

Let $M_{1}$ (resp. $\left.M_{2}\right)$ be an $m$ (resp. $\left.n-m\right)$-dimensional Riemannian manifold. An isometric immersion $f$ of a Riemannian product $M_{1} \times M_{2}$ into an $(n+p)$ dimensional Euclidean space $R^{n+p}$ is called a product immersion if there is an orthogonal product decomposition $R^{n+p}=R^{n_{1}} \times R^{n_{2}}$ together with isometric immersions $f_{1}: M_{1} \rightarrow R^{n_{1}}$ and $f_{2}: M_{2} \rightarrow R^{n_{2}}$ such that $f=f_{1} \times f_{2}$. Furthermore an isometric immersion $g$ of a Riemannian product $M_{1} \times M_{2}$ into an $(n+p)$-dimensional sphere $S^{n+p}(r)$ with radius $r$ in $R^{n+p+1}$ is called a product immersion if $g$ is a product immersion of $M_{1} \times M_{2}$ into $R^{n+p+1}$. S. B. Alexander [1] and J. Moore [4] obtained some conditions for an immersion of a Riemannian product into Euclidean space to be a product immersion. On the other hand, K. Yano and S. Ishihara [7] determined compact orientable submanifolds with nonnegative sectional curvature immersed into a unit sphere whose mean curvature vectors are parallel and normal connections are trivial. These are products of spheres and these immersions are product immersions into the unit sphere. In this note, we shall investigate Riemannian products minimally immersed into a real space form and prove some theorems.

THEOREM. A minimal submanifold of a hyperbolic space is irreducible. A minimal immersion of a Riemannian product into Euclidean space is a product of minimal immersions.

THEOREM. Let $M_{1}\left(\right.$ resp. $\left.M_{2}\right)$ be an $m($ resp. $n-m)$-dimensional compact orientable Riemannian manifold and $M$ the Riemannian product of $M_{1}$ and $M_{2}$ minimally immersed into $(n+p)$-dimensional unit sphere. Then we have an integral inequality

Received May 22, 1978 


$$
\int_{M}\left(n K-\lambda K-\mu K-\frac{2}{p} K^{2}\right) * 1 \geqq 0
$$

where $* 1$ is the volume element of $M$ and $\lambda$ (resp. $\mu$ ) is the minimum of the Ricci curvature of $M_{1}\left(\right.$ resp. $\left.M_{2}\right)$. The definition for $K$ is given in the section 5 .

By the latter theorem, we shall characterize a Riemannian product $S^{m}(1) \times S^{n-m}(1)$ minimally immersed into $S^{n+m(n-m)}(1)$.

The auther expresses his deep gratitude to Professor K. Ogiue who encouraged him and gave him a lot of valuable suggestions.

\section{§ 1. Preliminaries.}

We denote by $M^{k}(c)$ a $k$-dimensional space form of constant curvature c. Let $M_{1}$ (resp. $M_{2}$ ) be an $m$ (resp. $n-m$ )-dimensional Riemannian manifold and $M$ the Riemannian product of $M_{1}$ and $M_{2}$ isometrically immersed into $M^{n+p}(c)$. Then the second fundamental form $\sigma$ of the immersion is given by $\sigma(X, Y)=\tilde{V}_{X} Y-\nabla_{X} Y$ and it satisfies $\sigma(X, Y)=\sigma(Y, X)$. We choose a local field of orthonormal frames $e_{1}, \cdots, e_{m}$, (resp. $e_{m+1}, \cdots, e_{n}$ ) of $M_{1}$ (resp. $M_{2}$ ), then we may consider $\left\{e_{a}\right\},\left\{e_{s}\right\}$ as a local field of orthonormal frames of $M^{*}$. By an extension, we choose a local field of orthonormal frames $e_{1}, \cdots, e_{m}, e_{m+1}, \cdots, e_{n}, e_{n+1}, \cdots, e_{n+p}$ in $M^{n+p}(c)$. With respect to the frame field of $M^{n+p}(c)$ chosen above, let $\omega^{1}, \cdots, \omega^{m}, \omega^{m+1}, \cdots, \omega^{n}, \omega^{n+1}, \cdots, \omega^{n+p}$ be the field of dual frames. Then the structure equations of $M^{n+p}(c)$ are given by

$$
\begin{aligned}
& d \omega^{A}=-\sum \omega_{B}^{A} \wedge \omega^{B}, \quad \omega_{B}^{A}+\omega_{A}^{B}=0, \\
& d \omega_{B}^{A}=-\sum \omega_{C}^{A} \wedge \omega_{B}^{C}+c \omega^{A} \wedge \omega^{B},
\end{aligned}
$$

Restricting these forms to $M$, we obtain the structure equations of the immersion:

$$
\begin{gathered}
\omega^{\alpha}=0 . \\
d \omega^{i}=-\sum \omega_{j}^{i} \wedge \omega^{j}, \quad \omega_{j}^{i}+\omega_{i}^{j}=0 . \\
d \omega_{j}^{i}=-\sum \omega_{k}^{i} \wedge \omega_{j}^{k}+\Omega_{j}^{i}, \quad \Omega_{j}^{i}=\sum(1 / 2) R_{j k l}^{i} \omega^{k} \wedge \omega^{1} .
\end{gathered}
$$

* We use the following convention on the range of indices unless otherwise stated: $1 \leqq$ $A, B, C, \leqq n+p: 1 \leqq a, b, c, \leqq m<r, s, t, u, \leqq n: 1 \leqq i, j, k, l, \leqq n<\alpha, \beta, \gamma, \leqq n+p$, and agree that repeated indices under summation sign without any indication are summed over respective ranges. 


$$
d \omega_{\beta}^{\alpha}=-\sum \omega_{\gamma}^{\alpha} \wedge \omega_{\beta}^{\gamma}+\Omega_{\beta}^{\alpha}, \quad \Omega_{\beta}^{\alpha}=\sum(1 / 2) R_{\beta k l}^{\alpha} \omega^{k} \wedge \omega^{1} .
$$

Since $M$ is the Riemannian product, we have

$$
\omega_{s}^{a}=0 \text { for all } a \text { and } s \text {. }
$$

From (1.1) and (1.3) it follows that $\sum \omega_{i}^{\alpha} \wedge \omega^{i}=0$. Therefore, by Cartan's lemma, we may write

$$
\omega_{i}^{\alpha}=\sum h_{j i}^{\alpha} \omega^{j}, \quad h_{i j}^{\alpha}=h_{j i}^{\alpha} .
$$

The second fundamental form $\sigma$ and $h_{i j}^{\alpha}$ are related by $\sigma\left(e_{i}, e_{j}\right)=\sum h_{i j}^{\alpha} e_{\alpha}$. The equations of Gauss and Ricci are given respectively by

$$
\begin{gathered}
R_{j k l}^{i}=c\left(\delta_{k}^{i} \delta_{j l}-\delta_{l}^{i} \delta_{j k}\right)+\sum\left(h_{i k}^{\alpha} h_{j l}^{\alpha}-h_{i l}^{\alpha} h_{j k}^{\alpha}\right), \\
R_{\beta k l}^{\alpha}=\sum\left(h_{i k}^{\alpha} h_{i l}^{\beta}-h_{i l}^{\alpha} h_{i k}^{\beta}\right) .
\end{gathered}
$$

If we define $h_{i j k}^{\alpha}$ by

$$
\sum h_{i j k}^{\alpha} \omega^{k}=d h_{i j}^{\alpha}-\sum h_{k i}^{\alpha} \omega_{j}^{k}-\sum h_{k j}^{\alpha} \omega_{i}^{k}-\sum h_{i j}^{\beta} \omega_{\beta}^{\alpha} \text {. }
$$

Then from (1.2), (1.3), and (1.8) we have $h_{i j k}^{\alpha}=h_{i k j}^{\alpha}$. If we define $h_{i j k l}^{\alpha}$ by

$$
\sum h_{i j k l}^{\alpha} \omega^{1}=d h_{i j k}^{\alpha}-\sum h_{l i j}^{\alpha} \omega_{k}^{l}-\sum h_{l k j}^{\alpha} \omega_{i}^{l}-\sum h_{l i k}^{\alpha} \omega_{\jmath}^{l}+\sum h_{i j k}^{\beta} \omega_{\beta}^{\alpha},
$$

and $\Delta h_{i j}^{\alpha}$ by $\sum h_{i j k k}^{\alpha}$, then we have the following.

LEMMA 1.1 ([2], [5]). If $M$ is minimal,

$$
\Delta h_{i j}^{\alpha}=\sum\left(\sum h_{k l}^{\alpha} R_{i j k}^{l}+\sum h_{l i}^{\alpha} R_{k j k}^{l}-\sum h_{k i}^{\beta} R_{\beta j k}^{\alpha}\right)
$$

\section{§ 2. Lemmas.}

LEMmA 2.1 ([4]). When $c=0$, an immersion of $M=M_{1} \times M_{2}$ into $M^{n+p}(0)$ is a product immersion if and only if $\sigma(X, Y)=0$, where $X($ resp. $Y)$ is tangent to $M_{1}$ (resp. $\left.M_{2}\right)$.

LEMMA 2.2 ([8]). An immersion of $M=M_{1} \times M_{2}$ into $M^{n+p}(c)$ with $c>0$, is a product immersion if and only if $\sigma(X, Y)=0$, where $X($ resp. $Y)$ is tangent to $M_{1}\left(\right.$ resp. $\left.M_{2}\right)$.

Proof. We may regard $M^{n+p}(c)$ as the sphere $S^{n+p}(\sqrt{1 / c})$ of radius $\sqrt{1 / c}$ in $R^{n+p+1}$ and hence $M$ as a submanifold of $R^{n+p+1}$. Let $\tilde{\sigma}$ (resp. $\left.\sigma^{\prime}\right)$ be the second fundamental form of the immersion of $M$ (resp. $\left.S^{n+p}(\sqrt{1 / c})\right)$ into $R^{n+p+1}$. If $X(\operatorname{resp} . Y)$ is tangent to $M_{1}\left(\right.$ resp. $\left.M_{2}\right)$, then we have 
$\widetilde{\sigma}(X, Y)=\sigma(X, Y)$ since $\sigma^{\prime}$ is umbilical. From Lemma 2.1, we obtain the result.

Q.E.D.

Lemma 2.3. When $M=M_{1} \times M_{2}$ is minimally immersed into $M^{n+p}(c)$, we have $m(n-m) c-\sum_{\alpha}\left(\sum_{a} h_{a a}^{\alpha}\right)^{2}-\sum h_{a s}^{\alpha} h_{a s}^{\alpha}=0$.

Proof. Since $M$ is the Riemannian product of $M_{1}$ and $M_{2}$, we have $R_{s a s}^{a}=0$. From (1.9), we obtain $0=c+\sum_{\alpha} h_{a a}^{\alpha} h_{s s}^{\alpha}-\sum_{\alpha} h_{a s}^{\alpha} h_{a s}^{\alpha}$ and hence $0=$ $m(n-m) c+\sum_{\alpha}\left(\sum_{a} h_{a a}^{\alpha}\right)\left(\sum_{s} h_{s s}^{\alpha}\right)-\sum h_{a s}^{\alpha} h_{a s}^{\alpha}$. Since $M$ is minimally immersed into $M^{n+p}(c)$, we obtain $\sum_{a} h_{a a}+\sum_{s} h_{s s}=0$.

Q.E.D.

§3. In the case $c \leqq 0$.

THEOREM 3.1. (1) A minimal submanifold of $M^{n+p}(c)$ with $c<0$ is irreducible. (2) A minimal immersion of a Riemannian product into $M^{n+p}(0)$ is a product of minimal immersions.

Proof. (1) Lemma 2.3 implies that a submanifold minimally immersed into $M^{n+p}(c)$ with $c<0$ is irreducible. (2) We consider a Riemannian product of $M_{1}$ and $M_{2}$ minimally immersed into $M^{n+p}(0)$. From Lemma 2.3, we have $h_{a s}^{\alpha}=0$ which, together with Lemma 2.1, implies that the immersion is a product immersion. Moreover, by $\sum h_{a a}^{\alpha}=\sum h_{s s}^{\alpha}=0$, we see that each immersion is minimal.

Q.E.D.

REMARK. (1) More generally, a minimal submanifold immersed into a Riemannian manifold of negative curvature is irreducible. (2) Theorem 3.1 (2) holds for a Riemannian product of any number of Riemannian manifolds. (3) A Kähler immersion of a Riemannian product of Kähler manifolds into complex Euclidean space is a product of Kähler immersions.

§4. In the case $c>0$.

We may assume without loss of generality that $c=1$ and $M^{n+p}(1)=$ $S^{n+p}(1)$. When an immersion of $M=M_{1} \times M_{2}$ into $S^{n+p}(1)$ is a product immersion, we have some positive numbers $r_{1}, r_{2}$ such that $\left(r_{1}\right)^{2}+\left(r_{2}\right)^{2}=1$, positive integers $k_{1}, k_{2}$ such that $k_{1}+k_{2}+1=n+p$, and isometric immersions $f_{1}, f_{2}$ such that $f_{i}(i=1,2)$ is an immersion of $M_{i}$ into $S^{k_{i}}\left(r_{i}\right)$ and $I \circ\left(f_{1} \times f_{2}\right)$ is the immersion of $M$ into $S^{n+p}(1)$, where $I(x, y)=(x, y)$, where $x$ (resp. $y$ ) is the position vector of $S^{k_{1}}\left(r_{1}\right)\left(\right.$ resp. $\left.S^{k_{2}}\left(r_{2}\right)\right)$ in $R^{k_{1}+1}\left(\right.$ resp. $\left.R^{k_{2}+1}\right)$ and $(x, y)$ is the position vector of $S^{n+p}(1)$ in $R^{n+p+1}$. Immediately we have the following.

THEOREM 4.1. When an immersion of $M=M_{1} \times M_{2}$ into $S^{n+p}(1)$ is a 
product immersion, it is minimal if and only if $r_{1}=\sqrt{m / n}, r_{2}=\sqrt{(n-m) / n}$ and $f_{1}, f_{2}$ are minimal immersions.

In [2] and [6], we have some examples of Riemannian products minimally immersed into a unit sphere which are not product immersions. Some examples are:

EXAMPLE 1. Let $S^{m}(1)=\left\{\left(x_{1}, \cdots, x_{m+1}\right) \in R^{m+1} ;\left(x_{1}\right)^{2}+\cdots+\left(x_{m+1}\right)^{2}=1\right\}$ and $S^{n-m}(1)=\left\{\left(y_{1}, \cdots, y_{n-m+1}\right) \in R^{n-m+1} ;\left(y_{1}\right)^{2}+\cdots+\left(y_{n-m+1}\right)^{2}=1\right\}$. We define the immersion $f$ of $S^{m}(1) \times S^{n-m}(1)$ into $S^{n+m(n-m)}(1)$ by $f\left(\left(x_{1}, \cdots, x_{m+1}\right),\left(y_{1}, \cdots\right.\right.$, $\left.\left.y_{n-m+1}\right)\right)=\left(\cdots, x_{a} y_{s}, \cdots\right)$, where $1 \leqq a \leqq m, 1 \leqq s \leqq n-m$.

EXAMPLE 2. Let $M_{1}$ (resp. $\left.M_{2}\right)$ be an $m$ (resp. $\left.n-m\right)$-dimensional Riemannian manifold minimally immersed into $S^{k_{1}}(1)\left(\right.$ resp. $\left.S^{k_{2}}(1)\right)$. Then, by Example 1, the Riemannian product $M_{1} \times M_{2}$ can be immersed minimally into $S^{k_{1}+k_{2}+k_{1} k_{2}}(1)$.

We characterize $S^{m}(1) \times S^{n-m}(1)$ in Example 1 . We set $K=\sum h_{a s}^{\alpha} h_{a s}^{\alpha}$, which measures the deviation of the immersion from being product.

THEOREM 4.2. Let $M_{1}\left(\right.$ resp. $\left.M_{2}\right)$ be an $m($ resp. $n-m)$-dimensional compact orientable Riemannian manifold and $M$ be a Riemannian product of $M_{1}$ and $M_{2}$ minimally immersed into $S^{n+p}(1)$. Then we have an integral inequality,

$$
\int_{M}\left(n-\lambda-\mu-\frac{2}{p} K\right) K * 1 \geqq 0
$$

where $* 1$ is the volume element of $M$ and $\lambda(r e s p . \mu)$ is the minimum of the Ricci curvature of $M_{1}\left(\right.$ resp. $\left.M_{2}\right)$.

COROLlaRY 1. Under the same assumption as Theorem 4.2, if the immersion is full and $(n-\lambda-\mu-(2 / p) K) K \leqq 0$, then $K$ is constant and equal to 0 or $(p / 2)(n-\lambda-\mu)$. When $K=(p / 2)(n-\lambda-\mu) \neq 0, M$ is $S^{m}(1) \times$ $S^{n-m}(1)$ in Example 1 and the immersion is rigid.

CoROLlary 2. Under the same assumption as Theorem 4.2, if $\lambda+$ $\mu \geqq n$, then the immersion is a product immersion.

Proof of Theorem 4.2. $K$ is a function on $M$. Let $\Delta$ be the Laplacian of $M$. Then we obtain $(1 / 2) \Delta K=\sum h_{a s i}^{\alpha} h_{a s i}^{\alpha}+\sum\left(\Delta h_{a s}^{\alpha}\right) h_{a s}^{\alpha}$. From Lemma 1.1 we have

$$
\sum\left(\Delta h_{a s}^{\alpha}\right) h_{a s}^{\alpha}=\sum h_{a s}^{\alpha}\left(\sum h_{a i}^{\alpha} R_{j s j}^{i}+\sum h_{j i}^{\alpha} R_{a s i}^{j}+\sum h_{a i}^{\beta} R_{\alpha s i}^{\beta}\right)
$$

Since $M$ is a Riemannian product, $R_{a s i}^{j}=0$ and hence we have 


$$
\sum\left(\Delta h_{a s}^{\alpha}\right) h_{a s}^{\alpha}=\sum h_{a s}^{\alpha} h_{a i}^{\alpha} R_{j s j}^{i}+\sum h_{a s}^{\alpha} h_{a i}^{\beta} R_{\alpha s i}^{\beta} .
$$

Since the Ricci curvature of $M_{2} \geqq \mu$, we obtain

$$
\sum h_{a s}^{\alpha} h_{a i}^{\alpha} R_{j s j}^{\alpha}=\sum h_{a s}^{a} h_{a t}^{\alpha} R_{u s u}^{t} \geqq \mu \sum h_{a s}^{\alpha} h_{a s}^{\alpha}=\mu K,
$$

and hence

$$
\begin{aligned}
\sum\left(\Delta h_{a s}^{\alpha}\right) h_{a s}^{\alpha} & \geqq \mu K+\sum h_{a s}^{\alpha} h_{a i}^{\beta}\left(\sum h_{s j}^{\beta} h_{i j}^{\alpha}-\sum h_{i j}^{\beta} h_{s j}^{\alpha}\right) \\
& =\mu K+\sum h_{a s}^{\alpha} h_{i j}^{\alpha} h_{a i}^{\beta} h_{s j}^{\beta}-\sum h_{a s}^{\alpha} h_{s j}^{\alpha} h_{a i}^{\beta} h_{i j}^{\beta} .
\end{aligned}
$$

From (1.9), we have

$$
\begin{aligned}
\sum\left(\Delta h_{a s}^{\alpha}\right) h_{a s}^{\alpha} \geqq & \mu K+\sum h_{a s}^{\alpha} h_{i j}^{\alpha}\left(-R_{j s i}^{\alpha}+\delta_{s}^{\alpha} \delta_{j i}-\delta_{i}^{a} \delta_{j s}+\sum h_{a s}^{\beta} h_{j i}^{\beta}\right) \\
& -\sum h_{a s}^{\alpha} h_{s j}^{\alpha}\left(-R_{i j i}^{\alpha}+\delta_{j}^{\alpha} \delta_{i i}-\delta_{j}^{\alpha} \delta_{i j}+\sum h_{a j}^{\beta} h_{i t}^{\beta}\right) \\
= & \mu K+\sum h_{a s}^{\alpha} h_{i j}^{\alpha} h_{a s}^{\beta} h_{j i}^{\beta}+\sum h_{a s}^{\alpha} h_{s j}^{\alpha} R_{i j i}^{\alpha}-n K \\
\geqq & -n K+\lambda K+\mu K+\sum h_{a s}^{\alpha} h_{i j}^{\alpha} h_{s_{s}}^{\beta} h_{j i}^{\beta} \\
= & (\lambda+\mu-n) K+\sum h_{a s}^{\alpha} h_{b c}^{\alpha} h_{a s}^{\beta} h_{b c}^{\beta}+\sum 2 h_{a s}^{\alpha} h_{b t}^{\alpha} h_{a s}^{\beta} h_{b t}^{\beta} \\
& +\sum h_{a s}^{\alpha} h_{t u}^{\alpha} h_{a s}^{\beta} h_{t u}^{\beta} \geqq(\lambda+\mu-n) K+2 \sum h_{a s}^{\alpha} h_{b t}^{\alpha} h_{a s}^{\beta} h_{b t}^{\beta} .
\end{aligned}
$$

We set $S_{\alpha \beta}=h_{a_{s}}^{\alpha} h_{a_{s}}^{\beta}$. Then $\left(S_{\alpha \beta}\right)$ is a symmetric matrix. Therefore we have $\sum h_{a_{s}}^{\alpha} h_{b t}^{\alpha} h_{a_{8}}^{\beta} h_{b t}^{\beta}=\sum S_{\alpha \beta} S_{\alpha \beta} \geqq(1 / p)\left(\sum S_{\alpha \alpha}\right)^{2}=(1 / p)\left(\sum h_{a_{8}}^{\alpha} h_{a_{8}}^{\alpha}\right)^{2}=(1 / p) K^{2}$. The equality holds if and only if $\left(S_{\alpha \beta}\right)$ is proportional to the identity matrix. Since $M$ is compact and orientable, we obtain the integral inequality

$$
\int_{\mu}\left(n-\lambda-\mu-\frac{2}{p} K\right) K * 1 \geqq 0 \text {. }
$$

Proof of Corollaries 1 and 2. If $(n-\lambda-\mu-(2 / p) K) K \leqq 0$, we obtain $(n-\lambda-\mu-(2 / p) K) K=0$ and $K$ is constant. Furthermore we have

(1) $h_{a s i}^{a}=0$ for all $a, s, i$, and $\alpha$,

(2) $\sum h_{a_{s}}^{\alpha} h_{a_{s}}^{\beta}=0$ for all $\alpha \neq \beta$,

(3) $\sum_{a, s} h_{a s}^{\alpha} h_{a s}^{\alpha}=\sum_{a, s} h_{a s}^{\beta} h_{a s}^{\beta}$ for all $\alpha$ and $\beta$,

(4) $\sum h_{a_{s}}^{\alpha} h_{b c}^{\alpha}=0$ for all $a, b, c$, and $s$,

(5) $\sum h_{a_{s}}^{\alpha} h_{t u}^{\alpha}=0$ for all $a, s, t$, and $u$.

If we have $b_{0}$ and $c_{0}$ such that $\sigma\left(e_{b_{0}}, e_{c_{0}}\right) \neq 0$, we set $e_{n+1}=\left(\sigma\left(e_{b_{0}}, e_{c_{0}}\right)\right) /\left\|\sigma\left(e_{b_{0}}, e_{c_{0}}\right)\right\|$. we choose $e_{n+2}, \cdots, e_{n+p}$ so that $e_{n+1}, \cdots, e_{n+p}$ form a local field of normal frames. Then from (4) we have $h_{a s}^{n+1}=0$ and hence from (3) we have. $h_{a_{s}}^{\alpha}=0$ for all $\alpha$. So by Lemma 2.2 the immersion is a product immersion. Hence we assume the immersion is not a product immersion, i.e., $K \neq 0$. Then from the above discussion we have $\sigma\left(e_{b}, e_{c}\right)=0$, i.e., $h_{b_{0}}^{\alpha}=0$ for all $b, c$, and $\alpha$. Similarly we have $h_{s t}^{\alpha}=0$. This implies that $M$ is $S^{m}(1) \times S^{n-m}(1)$. We shall prove that the immersion is rigid. By the definition, 


$$
\sum h_{a b i}^{\alpha} \omega^{i}=d h_{a b}^{\alpha}-\sum h_{a i}^{\alpha} \omega_{b}^{i}-\sum h_{b i}^{\alpha} \omega_{a}^{i}+\sum h_{a b}^{\beta} \omega_{\beta}^{\alpha} .
$$

From $h_{a b b}^{\alpha}=0$ and (1.7), we obtain $h_{a b i}^{\alpha}=0$. Similarly $h_{s t i}^{\alpha}=0$. These, together with (1), imply that the second fundamental form $\sigma$ is parallel. From the assumption that the immersion is full and a result in [3], the normal space is spanned by the first normal space. Equation (1.9) shows that $\sum h_{a t}^{\alpha} h_{s b}^{\alpha}=\sum h_{a b}^{\alpha} h_{s t}^{\alpha}+\delta_{b}^{\alpha} \delta_{s t}$, which, together with $h_{a b}^{\alpha}=0$, implies $\sum h_{a t}^{\alpha} h_{s b}^{\alpha}=$ $\delta_{b}^{a} \delta_{s t}$. Thus if we set $e_{(a, s)}=\sigma\left(e_{a}, e_{s}\right)$, then $e_{(a, s)}, 1 \leqq a \leqq m<s \leqq n$, form a local field of normal frames, with respect to which $h_{b t}^{(a, s)}=\delta_{b}^{a} \delta_{t}^{s}$ holds. From (1) we have

$$
\mathbf{0}=\sum h_{b t i}^{(a, s)} \omega^{i}=d h_{b i}^{(a, s)}-\sum h_{b i}^{(a, s)} \omega_{t}^{i}-\sum h_{t i}^{(a, s)} \omega_{b}^{i}+\sum h_{b t}^{\beta} \omega_{\beta}^{(a, s)},
$$

which implies

$$
\omega_{(b, t)}^{(a, s)}=\delta_{b}^{a} \omega_{t}^{s}+\delta_{t}^{s} \omega_{b}^{a}
$$

So we have,

$$
\omega_{(b, t)}^{(a, s)}=0, \omega_{(a, t)}^{(a, s)}=\omega_{t}^{s} \text { and } \omega_{(b, s)}^{(a, s)}=\omega_{b}^{a},
$$

for all $a \neq b$ and $s \neq t$. Therefore the immersion is rigid.

Q.E.D.

For a result of [7], we have the following.

THEOREM 4.3. Let $M_{1}\left(\right.$ resp. $\left.M_{2}\right)$ be an $m($ resp. $n-m)$-dimensional compact and orientable Riemannian manifold and $M$ be a Riemannian product of $M_{1}$ and $M_{2}$ minimally immersed into $S^{n+p}(1)$. If the normal connection is trivial and either $M_{1}$ or $M_{2}$ has positive Ricci curvature, then the immersion is a product immersion.

Proof. We may assume that the Ricci curvature of $M_{2}$ is positive. Similar to the proof of Theorem 4.2, we have $\sum\left(\Delta h_{a s}^{\alpha}\right) h_{a s}^{\alpha} \geqq \mu K+\sum h_{a s}^{\alpha} h_{a i}^{\beta} R_{\alpha s i}^{\beta}$. Since the normal connection is trivial, we obtain $R_{\alpha s i}^{\beta}=0$. So we have $\sum\left(\Delta h_{a_{s}}^{\alpha}\right) h_{a_{s}}^{\alpha} \geqq \mu K$. Since $M$ is compact and orientable, we obtain $K=0$. From Lemma 2.2, the immersion is a product immersion. Q.E.D.

\section{References}

[1] S. B. Alexander, Reducibility of Euclidean immersions of low condimension, J. Differential Geometry, 3 (1969), 69-82.

[2] S. S. Chern, M. DoCarmo and S. Kobayashi, Minimal submanifolds of a sphere with second fundamental form of constant length, Functional Analysis and Related Fields, Springer, Berlin, 1970, 60-75.

[3] J. ERBACHER, Reduction of the codimension of an isometric immersion, J. Differential Geometry, 5 (1971), 303-340. 
[4] J. D. MOoRE, Isometric immersions of Riemannian products, J. Differential Geometry, 5 (1971), 159-168.

[5] J. Simons, Minimal varieties in Riemannian manifolds, Ann. of Math., 88 (1969), 62-105.

[6] N. R. WAllaCH, Minimal immersions of symmetric spaces into spheres, Symmetric Spaces, Marcel Dekker, New York, 1972, 1-40.

[7] K. YANO and S. IsHIHARA, Submanifolds with parallel mean curvature vector, J. Differential Geometry, 5 (1971), 95-118.

[8] S. T. YAU, Submanifolds with constant mean curvature II, Amer. J. Math., 96 (1975), 76-100.

Present Address:

Department of Mathematics TOKYo Metropolitan UNIVERSITY

Furazawa, Setagaya-ru, Tokyo 158 